\title{
Cost Effective Coverage Extension in IEEE802.16j Based Mobile WiMAX Systems
}

\author{
Se-Jin Kim, Byung-Bog Lee, Seung-Wan Ryu, \\ Hyong-Woo Lee and Choong-Ho Cho \\ Dept. of Computer and Information Science, Korea University, \\ Republic of Korea
}

\section{Introduction}

Recently, there have been numerous standardization activities for making the IEEE802.16e system highly effective in supporting mobile users based on orthogonal frequency division multiple access (OFDMA) with Time Division Duplex (TDD) mode in (IEEE Standard 802.16e-2005, 2005) and (WiMAX Forum). Standardization activities of the IEEE802.16e system were completed in 2005, and currently enhancements of the IEEE802.16e standard are under discussion. One such enhancement effort is cell coverage extension and link throughput enhancement which was studied in IEEE802.16j TG in (IEEE802.16j-2008, 2008). In order to achieve these goals, the IEEE802.16j TG introduces mobile multi-hop relay technology to the IEEE802.16e system. The other enhancement effort is being discussed in IEEE802.16m TG. The IEEE802.16m system, called the gigabit WiMAX, makinly aims to enhance system throughput up to $1 \mathrm{Gbps}$. Besides, this system aims to support legacy IEEE802.16 standards including the IEEE802.16j, and to interworking with other wireless systems such as 3GPP LTE and IMT-advanced system in (WiMAX Forum) and (Lee et al., 2006).

In particular, in Korea, the IEEE802.16e standard-based wireless broadband (WiBro) system was developed in 2005 and WiBro service was launched in 2006 covering isolated areas in Seoul. Since the initial launch, WiBro service providers have been trying to extend service areas from Seoul to regions nationwide. In order to cover the entire region of Korea, however, deployment cost for the traditional infrastructure consisting of only Base Stations (BSs) is estimated to be astronomical. For this reason, it is necessary to adopt a cost-effective service coverage extension method, and the mobile multi-hop relay (MMR) WiBro system is considered as a strong candidate for possible implementation.

In this chapter, we investigate various issues on multi-hop relay based WiBro/WiMAX systems, i.e., the IEEE802.16j system, with a focus on cost-effective cell coverage extension under various deployment situations. Since the coverage extension problem may occur in both of metropolitan areas and rural areas when the user-traffic density is relatively moderate or low, we first introduce an overview of IEEE802.16j standard. Secondly, we discuss several topologies and the resulting cost-effective coverage extension methods for 
each case. Also, we propose and analyze two sectored cellular based cost-effective coverage extension methods, the Narrow-Beam Trisector Cell (NBTC) and Wide-Beam Trisector Cell (WBTC) system based approaches. Finally, we present a practical deployment scenario consisting of three phases depending on the user-traffic density and the number of traffic relaying hops.

The remainder of this chapter is organized as follows. In section 2, we introduce standardization and research issues in MMR WiBro/WiMAX system. In section 3, we analyze cost-effective coverage extension methods under various MMR topologies that may occur in metropolitan and rural areas. Then we present sectored cellular-based coverage extension approaches in section 4 . Based on the above analysis results, we propose a practical deployment scenario in section 5. Finally, we conclude this chapter by suggesting future research issues in section 6.

\section{An overview of the MMR WiBro/WiMAX system}

\subsection{Standardization and research issues in the MMR WiBro/WiMAX system}

In general, the concept of multi-hop relaying is adopted to extend cell coverage, to enhance link throughput per user, and to increase network reliability, while supporting quality of service requirements. One approach to enhance system capacity, throughput per user, and system reliability is adoption of Relay Stations (RSs) in low SINR cell boundary areas. In addition, by using demodulation/decode and forward functionalities, RSs could substitute higher data rate SINR links for existing lower SINR links. This spectrum efficiency results in system capacity enhancement. In fact, a part of the enhanced capacity could be used for multi-hop relay function while the other part of the capacity could be used to provide subscribers with increased throughput.

The purpose of cell-coverage extension is three-fold. First, it can be used to remove coverage holes that can be found around high-rise buildings or other wave propagation obstacles. Second, it can be used to give safe communication coverage to subscribers who are far away from or isolated from a BS. Third, it can be used to provide reliable communication links to mobile vehicles on which an RS is installed.

IEEE802.16j TG aims to enhance the IEEE802.16e (mobile WiMAX or WiBro) system in terms of throughput per user and cell coverage by introducing multi-hop based RSs in (IEEE 802.16j-2008, 2008) and (Siemens and ETRI, 2007). Coverage extension in the legacy WiBro/WiMAX system can be achieved based on the existing PMP (Point to Multi-Point) mode, while throughput enhancements can be achieved by higher-order modulation schemes based on the higher quality signal strength between BS and RS, and RS and Mobile Station (MS). However, without introducing MMR technology, even all the enhancements, which are related to the PHY (Physical) and the MAC (Medium Access Control) layers, are restricted to two hops, from BS to RS and RS to MS.

Fig.1 shows the overall MMR system's configuration studied in IEEE802.16j TG. The RS being considered in IEEE802.16j TG can be categorized into 3 types: the fixed RS, the nomadic RS, and the mobile RS. In addition, direct communication between MSs is not allowed. Therefore, the full mesh-networking approach support connections and multiple hops are beyond the scope of IEEE802.16j TG in (IEEE802.16j-2008, 2008). 


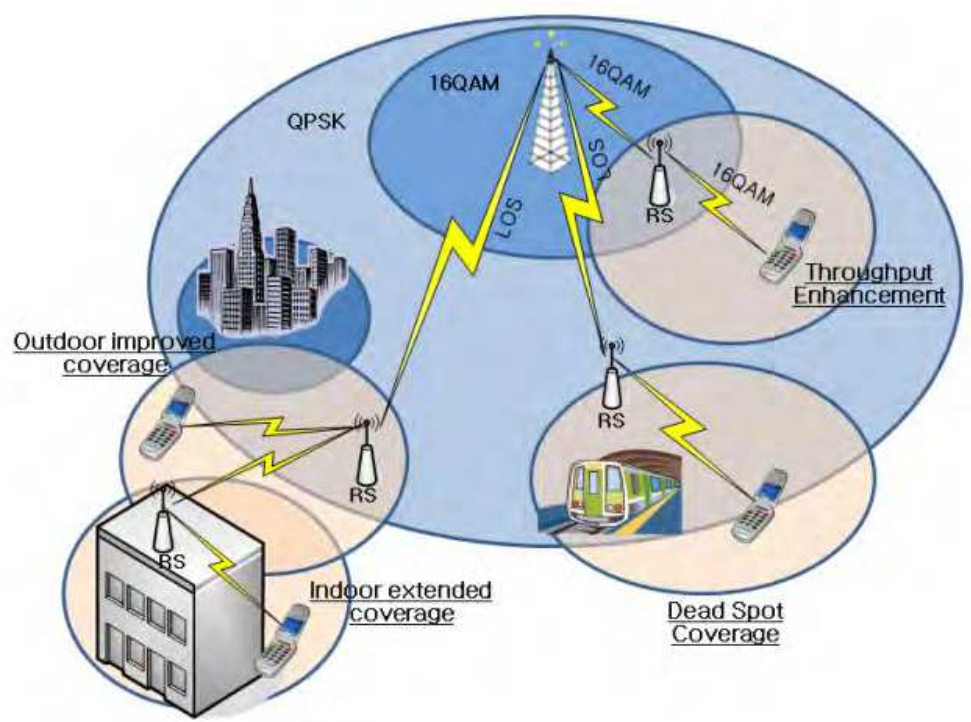

Fig. 1. IEEE802.16j TG MMR system.

\subsection{Technical requirements for relay stations of the MMR WiBro/WiMAX}

There are several technical requirements for the RSs of the MMR WiBro/WiMAX system (Siemens and ETRI, 2007). In this subsection, we summarize major technical functionalities of RSs to improve network throughput and to extend cell coverage.

- Support of legacy WiBro/WiMAX services: An RS should be able to support WiBro/WiMAX services to MSs transparently. An RS should register its existence to the network, and relay uplink and downlink traffic between the BS and an MS based on the WiBro/WiMAX protocols in (Lee et al., 2006) and (Siemens and ETRI, 2007).

- Network Entry functionalities: An RS could be operated in both MS-mode and BSmode. When an RS is turned on, the RS can be operated in MS-mode to obtain synchronization information from the BS. After the synchronization process, the RS negotiates with the BS for the capability of an RS. Then, the RS operates in MS-mode for communication with the BS, and in BS-mode for communication with MSs. Therefore, the RS should include both MS-mode and BS-mode functionalities.

- Handover Support: Since an RS must provide legacy WiBro/WiMAX services, it should be able to support MS mobility. RS handover procedures that are similar to MR-BS handoff procedures should be performed for the usage models which can be found in guideline document in (IEEE802.16j-06/015, 2006).

- Cell-throughput enhancement: In order to enhance cell throughput per user, an RS should support Adaptive Modulation Coding (AMC) technology in hop-by-hop links, such as relay links and access links. Also cooperative-relay technology is considered to achieve cell-throughput enhancement.

- Cell-coverage extension: In order to extend cell coverage by multi-hop links, RSs should relay the traffic from MR-BS/RS to RS/MS and vice versa. There could be 
several kinds of frame structures that can support such multi-hop links. A transparent frame structure and a non-transparent frame structure were adopted in IEEE802.16j standard.

\subsection{Transparent mode relaying vs. non-transparent mode relaying}

In IEEE802.16j TG standardization, two types of RS modes, the transparent mode and the non-transparent mode, are proposed for the TDD mode. The key difference between these two relay modes is the method of transmitting the framing information.

In transparent mode, the RSs do not forward the frame header information, and hence do not extend the cell coverage of the wireless access system. Consequently, the main use case for transparent mode RSs is to facilitate increases in capacity within the cell coverage. This type of RS is of lower complexity, and only operates in a centralized scheduling mode for a topology of up to two hops.

On the other hand, the non-transparent mode RS is generally located in the cell edge to extend BS coverage using the remaining capacity of the BS. The RSs generate their own framing information or forward those provided by the BS depending on the scheduling approach (i.e., distributed or centralized). In the centralized scheduling, RSs relay MAP information received from the BS to its neighboring offspring RSs and MSs. Therefore, the centralized scheduling scheme is simple and efficient, and thus used in smaller grids such as metropolitan-area topologies. Meanwhile, in the distributed scheduling, an RS receives MAP information from the BS similar to the centralized mode. However, it creates its own MAP information and transmits it to its neighboring offspring RSs and MSs. Therefore, the distributed scheduling scheme can provide fault tolerance and scalability for large-scale topologies. However, the transmission of the framing information can result in high interference between neighboring RSs. Also, non-transparent mode RSs can operate in topologies larger than two hops in either centralized or distributed scheduling mode.

As with the former 802.16e frame structure in (IEEE802.16e-2005, 2005), the 802.16j frame is divided into the DL and UL subframes. However, unlike the previous frame structure, these subframes are further divided into zones to support BS-RS/MS communication (the Access Zone (AZ)) and RS-MS communication (the Relay Zone (RZ)). Thus, the Relay Receive/transmit Transition Interval (R-RTI) and Relay Transmit/receive Transition Interval (R-TTI), have to be inserted between AZ and RZ for both the DL and UL subframes in order to give the wireless devices sufficient time to switch from transmission mode to reception mode, or vice versa.

In transparent mode, the BS transmits data to both of the MSs and RSs in AZ, and then the RSs retransmit the received data to MSs located in its coverage. In RZ, the BS operates either in a silent mode or in a cooperative transmission mode. The frame structure of the nontransparent mode is similar to that of the transparent mode, but a BS and RSs can transmit data at the same time in AZ due to lower interference. An RS retransmits the received data to MSs located in its coverage. In RZ, a BS transmits data to RSs and all RSs operate in receiving mode.

The coverage extension, therefore, can be achieved using the non-transparent mode RSs. For the remainder of this chapter, we will concentrate on the non-transparent mode RSs. 


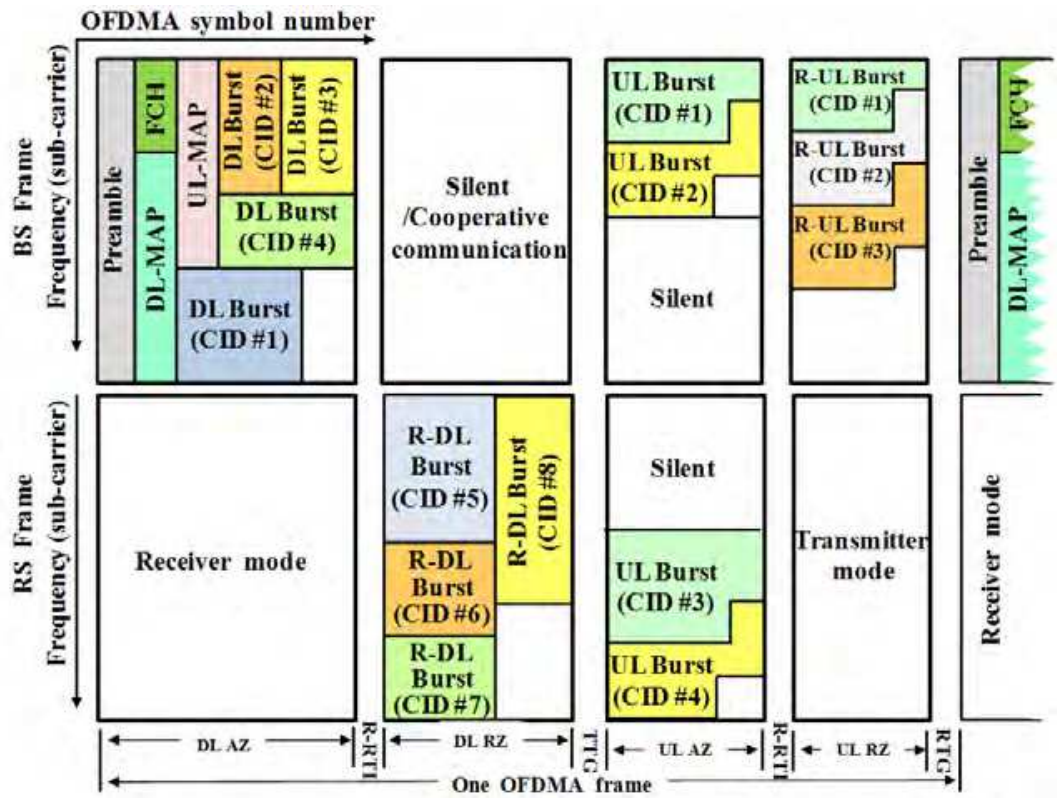

(a) Transparent mode

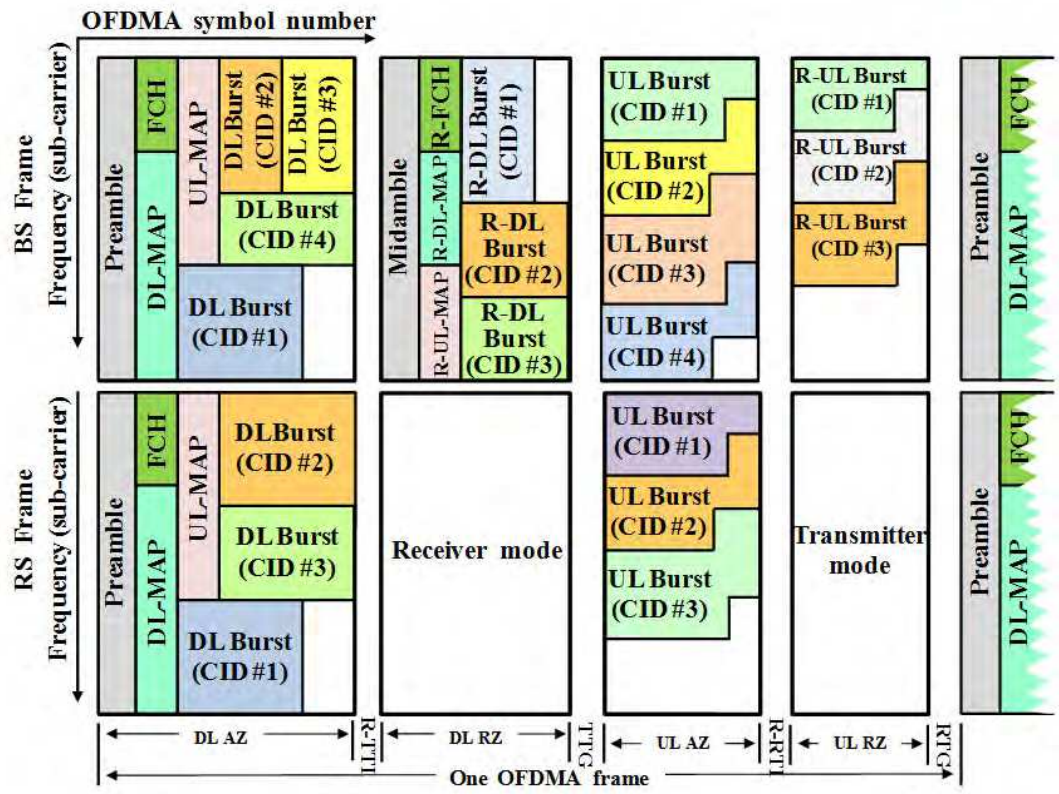

(b) Non-transparent mode

Fig. 2. Frame structures in IEEE802.16j. 


\section{Coverage extension with minimal deployment cost}

In this section, we propose and analyze various MMR topologies for cell-coverage extension using omni-directional antennas. Under such topologies, we investigate deployment cost and optimal numbers of BSs and RSs with respect to various user traffic densities.

\subsection{Omni-directional antenna based multi-hop relay topologies}

We propose various MMR topologies for both metropolitan and rural areas. We assume that omni-directional antennas are used in both a BS and RSs. We also assume that the maximum number of hops is 4 which means that there could be at most 3-tiers of RSs from the BS. For the convenience of analysis, we also assume that the unit coverage areas in the metropolitan area topologies and the rural area topologies are a square and a hexagon respectively. A BS is assumed to have different sizes of coverage area according to its transmission power. In other words, in terms of the unit coverage area, a BS could have a different number of unit areas for its coverage. In general, RSs have less transmission power compare to the BS, and thus, in this chapter, we assume that each RS covers one unit of coverage area regardless of topologies. Figure 3 shows three types of different topologies for each case of the metropolitan and rural areas, i.e., Type-A, B, and C, for the metropolitan areas and Type-D, E, and F are for the rural areas. For the metropolitan areas, we use grid topology models which are generally used for metropolitan topology. An RS coverage is a square but the BS coverages are one, four, five squares for Type-A, B, C. On the other hand, for the rural areas, we use hexagonal topology models. An RS coverage is a hexagon but the BS coverages are one, seven, three hexagons for Type-D, E, F. The fill patterns and colors denote the number of tiers for RSs.

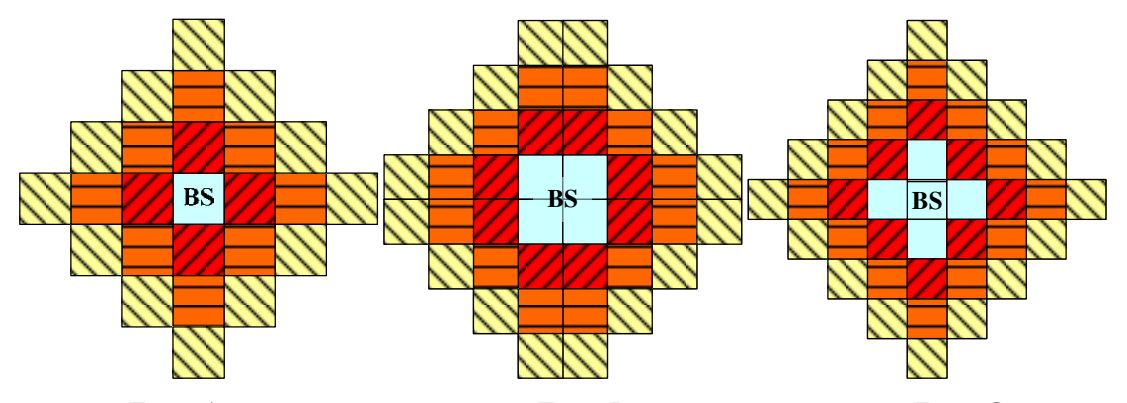

Type-A

Type-B

Type-C

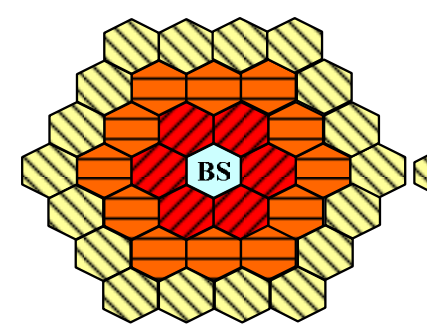

Type-D
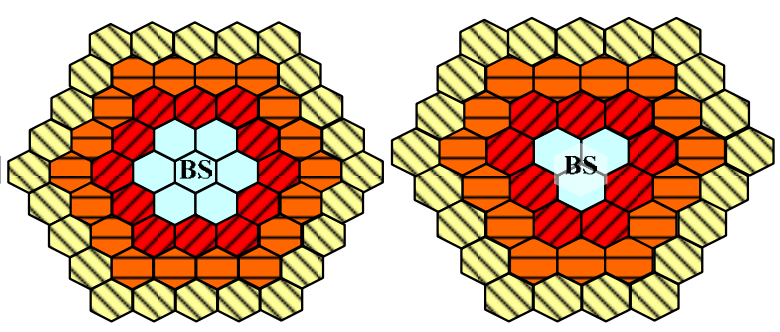

Type-F

Fig. 3. Multihop-based topologies in metropolitan (Type-A, B, and C) and rural (Type-D, E, and F) area. 


\subsection{Modeling of a cost-effective coverage extension problem}

When a target service area is given, the cost-effective coverage enhancement problem of the MMR WiBro/ WiMAX system is generally solved with respect to the traffic density per unit area. Since each BS and RS has its own coverage area in terms of unit service area and the user traffic is generated proportional to the size of service area, each BS and RS has a maximum number of accommodating MSs for its coverage area. In addition, since a BS has limited capacity, a limited number of RSs can be connected to a BS for coverage extension. From the characteristics of each proposed MMR topology, the number of RSs in each tier is also limited. For example, in case of type B, there could be at most 8, 12, or 18 RSs in each tier, respectively.

As a result, the cost-effective coverage extension problem can be formulated as a cost minimization problem with several constraints for a given user-traffic density using an integer Linear programming. Then, it can be expressed in terms of the number of BSs and RSs that are required to cover the target service area for a given user-traffic density. The object function and constraints for the minimum deployment cost are evaluated by the following optimization problem:

Minimize

$$
C_{T}=C_{B S} X_{B S}+C_{R S} X_{R S \_1}+C_{R S} X_{R S \_2}+C_{R S} X_{R S \_3}
$$

Subject to

$$
\begin{aligned}
& A_{B S} X_{B S}+A_{R S} X_{R S \_1}+A_{R S} X_{R S \_2}+A_{R S} X_{R S \_3} \geq A_{T} \\
& \left(C-\rho^{*} A_{B S}\right) X_{B S}-\rho^{*} A_{R S} X_{R S \_1} \\
& -\rho^{*} A_{R S} X_{R S \_2}-\rho^{*} A_{R S} X_{R S \_3} \geq 0 \\
& X_{R S_{-} 1}-N_{1} \cdot X_{B S} \leq 0 \\
& N_{1} \cdot X_{R S_{-} 2}-N_{2} \cdot X_{R S_{-} 1} \leq 0 \\
& N_{2} \cdot X_{R S_{-} 3}-N_{3} \cdot X_{R S_{-} 2} \leq 0 \\
& X_{B S} \in\{1,2, \cdots\} \\
& X_{R S_{-} j} \in\{0,1,2, \cdots\}, j \in\{1,2,3\}
\end{aligned}
$$

where, $C_{T}$ is total deployment cost for a given user traffic density, $X_{B S_{-} i}$ is the number of BSs and $X_{R S_{-} i}$ is the number of RSs at $i$-th tier. $C_{B S}$ and $C_{R S}$ are the costs of a BS and an RS, respectively. There are four constraints in the optimization problem; constraints for seamless covering of the target service area in (2) where $A_{B S}$ and $A_{R S}$ are the coverages of a BS and an RS, constraints for the capacity limitation of a BS in (3) where $C$ is the capacity of a BS and $\rho$ is a given user traffic density, constraints for the maximum number of RSs $\left(N_{1} \sim N_{3}\right)$ at each tier in (4), and constraints for the integrality of the number of BSs and RSs in (5) and (6). 


\subsection{Performance analysis of coverage extension and deployment cost}

In order to analyze the cost-effective coverage extension problem for each topology under a given user-traffic density, several modeling parameters shown in Table 1 are used. We used CPLEX for optimization tool. In the analysis, for the sake of modeling convenience, it is assumed that user traffic is uniformly distributed, no Adaptive Modulation Coding (AMC) option, no resource allocation for BS and RSs, and no interference models are used. In addition, the cost of an RS is assumed to be a portion of the cost of a BS. In this chapter, three different RS cost levels, $10 \%, 20 \%$ and $30 \%$ of a BS cost, are used

\begin{tabular}{|c|c|c|c|c|}
\hline \multirow{2}{*}{ Parameter } & \multicolumn{4}{|c|}{ Value } \\
\hline \multirow{2}{*}{ Total area $\left(A_{T}\right)$} & \multicolumn{3}{|c|}{$300 \mathrm{Km}^{2}$} \\
\hline \multirow{2}{*}{ BS coverage $\left(A_{B S}\right)$} & Metropolitan Area & \multicolumn{2}{c|}{ Rural Area } \\
\cline { 2 - 5 } & Type-A & $1 \mathrm{Km}^{2}$ & Type-D & $1 \mathrm{Km}^{2}$ \\
\cline { 2 - 5 } & Type-B & $4 \mathrm{Km}^{2}$ & Type-E & $7 \mathrm{Km}^{2}$ \\
\cline { 2 - 5 } & Type-C & $5 \mathrm{Km}^{2}$ & Type-F & $3 \mathrm{Km}^{2}$ \\
\hline \multirow{2}{*}{ RS coverage $\left(A_{R S}\right)$} & $1 \mathrm{Km}{ }^{2}($ a square $)$ & $1 \mathrm{Km}{ }^{2}(\mathrm{a}$ hexagon $)$ \\
\hline Traffic density $(\rho)$ & \multicolumn{3}{|c|}{$0.1 \sim 10 \mathrm{bps} / \mathrm{m}^{2}\left(0.1 \sim 10 \mathrm{Mbps} / \mathrm{km}^{2}\right)$} \\
\hline RS cost $\left(C_{R S}\right)$ & \multicolumn{4}{|c|}{$10 \%, 20 \%, 30 \%$ of BS cost per RS } \\
\hline BS total capacity $(C)$ & \multicolumn{3}{|c|}{$50 \mathrm{Mbps}$} \\
\hline
\end{tabular}

Table 1. Model Parameters for Metropolitan and Rural area systems.

Figure 4 shows the efficiency of the MMR-based coverage extension approach in terms of the coverage extension and the total deployment cost for the case of metropolitan areas and rural areas, respectively. Figure 4-(a) shows that the coverage area with the type-C approach is bigger than with type-A when the same number of BSs is used. However, as the traffic density increases, the difference between each MMR approach and the difference between MMR approaches and BS-only approach tend to vanish due to the limited capacity of a BS. Figure 4-(b) shows the total deployment cost of three MMR approaches and BS-only approaches for seamless covering of the target metropolitan service area with respect to the traffic density. As shown in Figure 4-(b), type-C topology covers the target area with the lowest cost while type-A covers with the highest cost. The reason is that because of small coverage of a BS, type-A requires more number of BSs to cover the target area, and thus results higher deployment cost. Meanwhile, when RS costs of type-B and type-C are 30\%, and $20 \%$ and $30 \%$ of BS cost, deployment of such MMR topologies are not beneficial than the BS-only approach because of the higher RS cost compared to the coverage extension effect. 


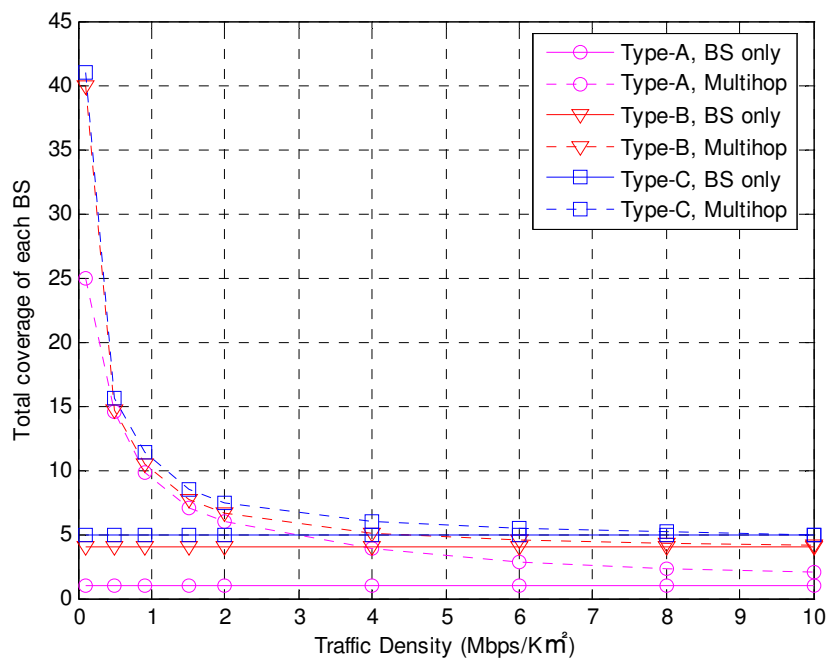

(a) coverage extension

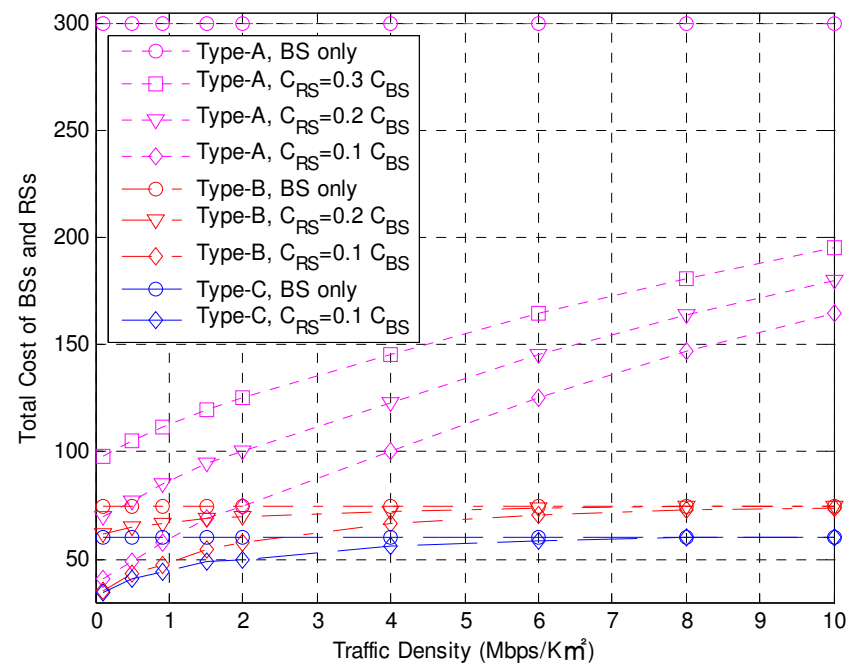

(b) Deployment cost

Fig. 4. Analysis results on coverage extension (a) and deployment cost (b) under various topologies for metropolitan area.

Figure 5-(a) also shows that the coverage area with type-E approach is bigger than with type-D when the same number of BSs is used. However, similar to the result of figure 4-(a), as the traffic density increases, the difference between each MMR approach types and the difference between MMR approaches and BS-only approach are tend to vanish due to the limited capacity of a BS. Figure 5-(b) shows the total deployment cost of three MMR approaches and 
BS-only approaches for seamless covering of the target rural service area with respect to the traffic density. As shown in Figure 5-(b), type-E topology covers the target area with the lowest cost while type-D covers with the highest cost due to the same reason explained in figure 4-(b). In addition, similar to the figure $4-(\mathrm{b})$, when RS costs of type-E are $20 \%$ and $30 \%$ of BS cost, deployment of such MMR topologies are not beneficial than the BS only approach because of the same reason explained in figure 4-(b).

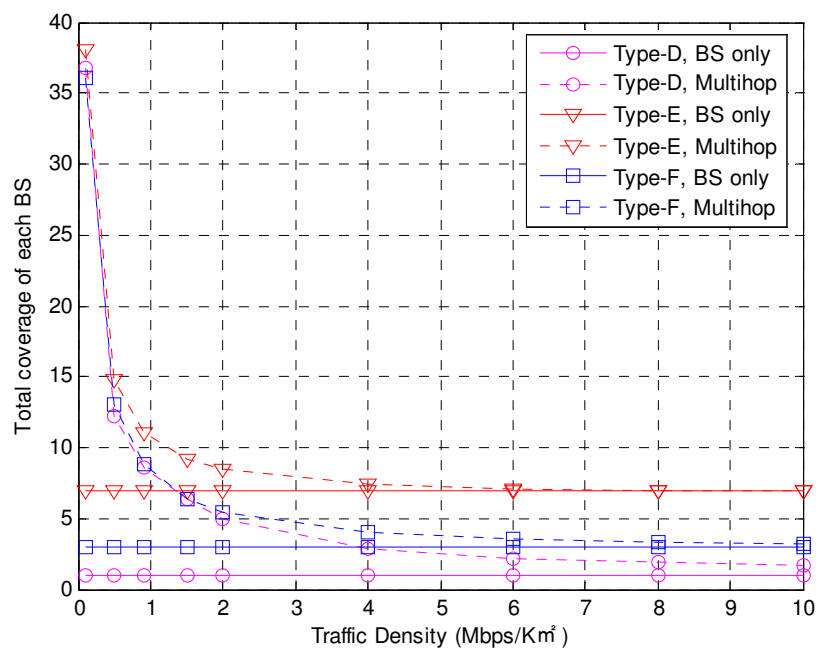

(a) coverage extension

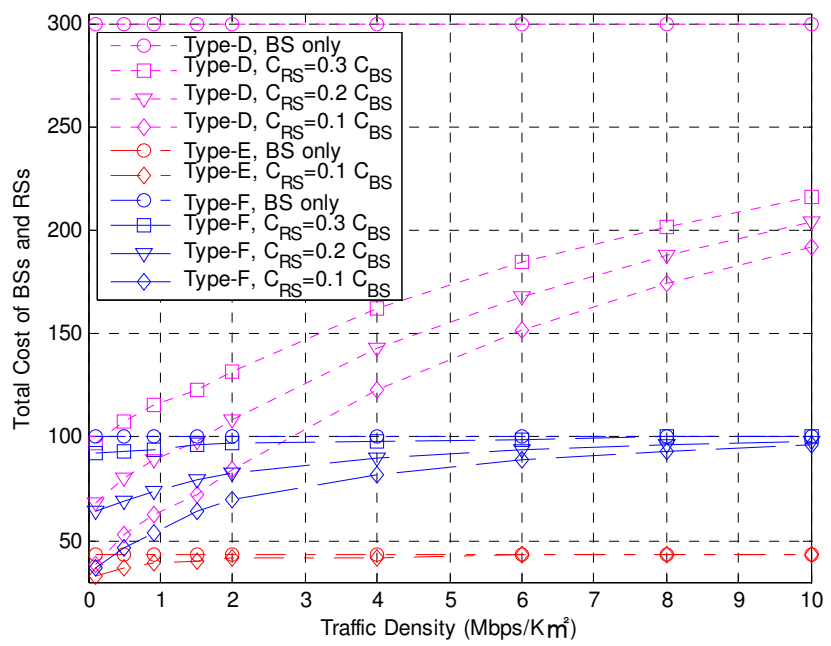

(b) Deployment cost

Fig. 5. Analysis results on coverage extension (a) and deployment cost (b) under various topologies for rural area. 


\section{Coverage extension with sectorized approaches}

In this section, we propose and analyze various MMR topologies for cell-coverage extension using directional-antennas. Under such topologies, we investigate deployment cost and optimal numbers of BSs and RSs with respect to various user traffic densities.

\subsection{Sectored BS based Multi-hop relay approaches}

In the conventional cellular system with omni-directional antenna, the contour of a cell for equal received power from the antenna is approximately a circular, and generally assumed

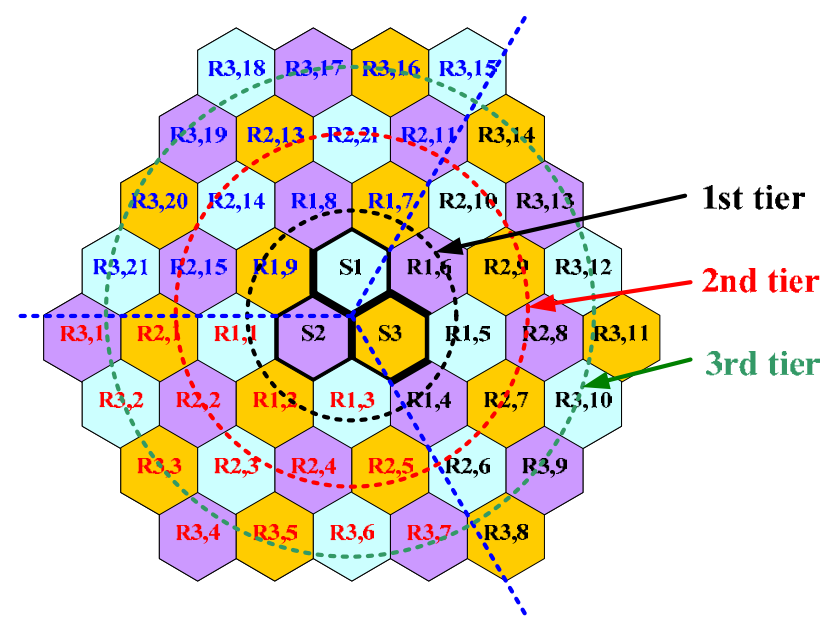

(a) Multihop-based NBTC

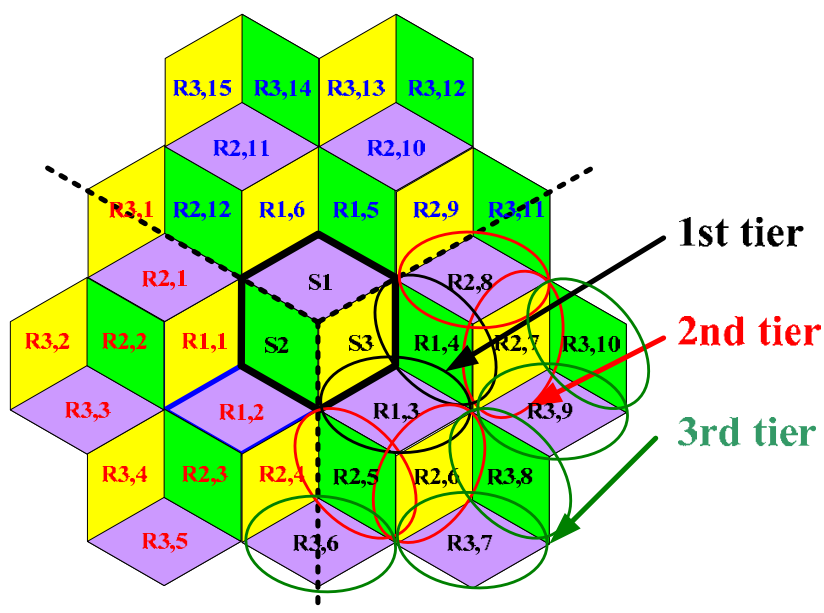

(b) Multihop-based WBTC

Fig. 6. Multihop-based NBTC and WBTC systems. 
to be a hexagonal shape in (IEEE 802.16j-06/015, 2006). Consequently, in order to increase a BS capacity through decreasing radio frequency interference, sectorized cell structures such as the WBTC and NBTC systems were introduced in (Noh, 2006) and (Choi et al., 2006). The number of sectors in a cell is depend on the characters of antenna but three sectored $\left(120^{\circ}\right)$ or six sectored $\left(60^{\circ}\right)$ antenna is normally used. In general, the representative three sector cell structures are the WBTC and NBTC. A BS is covered with three $60^{\circ}$ directional antennas in the NBTC. The coverage area of a sector in the NBTC assumed to be a hexagonal shape because the narrow beam radiation pattern matches well a hypothetical hexagonal shape. With three such antennas, the coverage contour of the NBTC cell composed of three sectors is therefore like a clover leaf. On the other hand, the coverage area of a sector in the WBTC assumed to be a rhombus shape because the wide beam radiation pattern with $120^{\circ}$ antennas matches well a rhombus shape, and thus the coverage area of the WBTC is a hexagonal shape consisting of three rhombus shape sectors.

We propose two multi-hop approaches based on NBTC and WBTC BS sectorization. Figure 6 shows two multi-hop relay approaches based on the NBTC and WBTC BS structures. As shown in figure 6-(a), an NBTC BS is assumed to have three hexagonal shape sectors $\left(S_{i}\right.$, $i=1,2,3)$, and the shape of each RS is assumed to be a hexagon. On the other hand, as shown in figure 6-(b), a WBTC BS is assumed to have three rhombus shape sectors $\left(S_{i}, i=1,2,3\right)$, and the shape of each RS is assumed to be a rhombus.

In this chapter, we only consider the NBTC and the WBTC based MMR approaches having up to 3 tiers. $S_{i}$ means $i$-th sector of a BS and $R_{i, j}$ means the $j$-th RS at $i$-th tier. For example, every sector in NBTC based MMR system may have at most three RSs at the first tier, five RSs at the second tier, and seven RSs at the third tier as illustrated in figure 6-(a). On the other hand, every sector in WBTC based MMR system may have at most two RSs at the first tier, four RSs at the second tier, and five RSs at the third tier as illustrated in figure 6-(b).

\subsection{Problem formulation and performance analysis}

In order to analyze the cost-effective coverage extension problem for each sectorized BS based MMR approaches under a given user traffic density, we formulate similar integer Linear optimization problem introduced in the previous section where omni-directional antenna is assumed to use. The object function of the sectorized BS based MMR approach is slightly different from the previous model. In the sectorized BS based MMR approach, the BS cost is divided into two parts, one for BS tower construction cost and the other for sector antenna related cost. Therefore, the objective function and constraints can be expressed as below.

Minimize

$$
\begin{aligned}
C_{T} & =C_{B S} X_{B S}+C_{R S} \sum_{i=1}^{3} \sum_{j=1}^{l} X_{R S_{i, j}} \\
& =C_{B S}^{*} X_{B S}+\sum_{i=1}^{3} C_{B S}^{i} X_{R S_{i, 0}}+C_{R S} \sum_{i=1}^{3} \sum_{j=1}^{l} X_{R S_{i, j}}
\end{aligned}
$$

Subject to 


$$
\left.\begin{array}{c}
A_{B S} X_{B S}+A_{R S} \sum_{i=1}^{3} \sum_{j=1}^{l} X_{R S_{i, j}} \geq A_{T} \\
C \cdot X_{B S}-\rho\left(\sum_{i=1}^{3} \sum_{j=0}^{l} X_{R S_{i, j}}\right) \geq 0 \\
X_{R S_{i, 0}}-N_{1} \cdot X_{B S}^{i} \leq 0, \\
N_{1} \cdot X_{R S_{i, 2}}-N_{2} \cdot X_{R S_{i, 1}} \leq 0, \\
N_{2} \cdot X_{R S_{i, 3}}-N_{3} \cdot X_{R S_{i, 2}} \leq 0, \\
N_{3} \cdot X_{R S_{i, 4}}-N_{4} \cdot X_{R S_{i, 3}} \leq 0,
\end{array}\right\} \begin{aligned}
& f o r \forall i \in\{1,2,3\} \\
& X_{B S}-\left(X_{R S_{1,0}}+X_{R S_{2,0}}+X_{R S_{3,0}}\right) \leq 0, \\
& 3 X_{B S}-\left(X_{R S_{1,0}}+X_{R S_{2,0}}+X_{R S_{3,0}}\right) \geq 0, \\
& X_{R S_{i, j}} \in\{0,1,2, \cdots\} \text { for } \forall i \in\{1,2,3\}, \forall j \in\{0,1,2,3, \cdots\} \\
& X_{B S}=X_{R S_{1,0}} \Rightarrow\left\{\begin{array}{l}
X_{B S}-X_{R S_{1,0}} \leq 0, \\
X_{B S}-X_{R S_{1,0}} \geq 0,
\end{array}\right. \\
& X_{R S_{1,0}} \geq X_{R S_{2,0}} \geq X_{R S_{3,0}} \Rightarrow\left\{\begin{array}{l}
X_{R S_{1,0}}-X_{R S_{2,0}} \geq 0, \\
X_{R S_{2,0}}-X_{R S_{3,0}} \geq 0
\end{array}\right.
\end{aligned}
$$

where, $C_{T}, C_{B S}$ and $C_{R S}$ are the same cost values used in (1), and $X_{B S}$ is the number of BS. In order to consider sectorized BS based MMR approach, several variables are introduced. $X_{R S_{i, j}}$ is the number of RS at $\mathrm{i}$-th tier of $\mathrm{j}$-th BS sector, and $X_{R S_{i, 0}}$ is the number of sector antenna in BSs. C*BS is the BS tower construction costs, and CiBS is the $\mathrm{i}$-th sector antenna related cost in a BS. Constraints of the optimization problem and assumptions are similar to the previous optimization model in sub-section 3.2. The constraint for seamless covering of the target service area is in (8) where AiBS is the coverage of a directional-antenna in a BS and (9) is the constraints for the capacity limitation of a BS. (10-1) is the constraints for the maximum number of RSs at each tier where N1 N4 are variables and (10-2) is the constraint about that a BS can have three directional-antennas. (10-3) is the constraint about a BS must have a sectored antenna and (10-4) is the order of sectored antennas in a BS. The constraints of (11) and (12) are the integrality of the number of BSs and RSs.

Figure 7 shows analytical results on coverage extension and deployment cost for NBTC and WBTC BS-based MMR systems. Modeling parameters are the similar to the previous model shown in Table 2. 


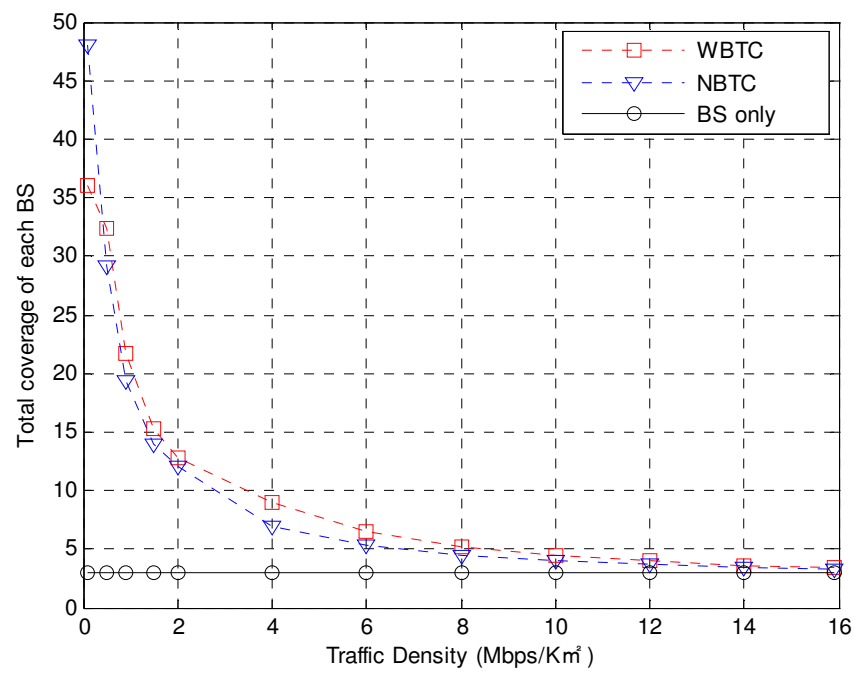

(a) coverage extension

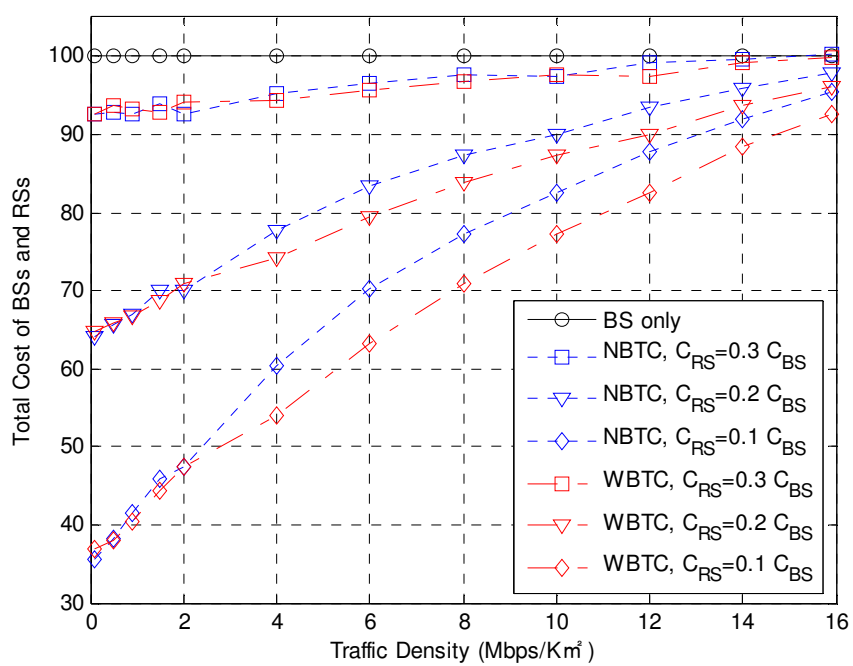

(b) Deployment cost

Fig. 7. Analysis results on coverage extension (a) and deployment cost (b) under WBTC and NBTC based MMR systems. 


\begin{tabular}{|c|c|}
\hline Parameter & Value \\
\hline Total area $\left(A_{T}\right)$ & $300 \mathrm{Km}^{2}$ \\
\hline BS coverage $\left(A_{B S}\right)$ & $\begin{array}{c}3 \mathrm{Km}^{2} \\
\text { (Three sector antennas) }\end{array}$ \\
\hline RS coverage $\left(A_{R S}\right)$ & $1 \mathrm{Km}^{2}$ \\
\hline Traffic density $(\rho)$ & $\begin{array}{c}0.1 \sim 15.9 \mathrm{bps} / \mathrm{m}^{2} \\
\left(0.1 \sim 15.9 \mathrm{Mbps} / \mathrm{km}^{2}\right)\end{array}$ \\
\hline RS cost $\left(C_{R S}\right)$ & $10 \%, 20 \%, 30 \%$ of BS cost per RS \\
\hline BS total capacity $(C)$ & $\begin{array}{c}60 \mathrm{Mbps} \\
(\text { Each antenna has } 20 \mathrm{Mbps})\end{array}$ \\
\hline
\end{tabular}

Table 2. Model Parameters for NBTC and WBTC Systems.

Figure 7 shows the analytical results of coverage extension and deployment cost for the NBTC and the WBTC BS based MMR systems with respect to the user traffic density under $300 \mathrm{Km}^{2}$ of the target service area. As shown in figure 7-(a), the coverage of the WBTC BSbased MMR system is shown to be slightly larger than that of the NBTC BS-based MMR system while, the coverage of the NBTC BS-based MMR system is shown to be larger than that of the WBTC BS-based MMR system when the traffic density is less than 0.4 $\mathrm{Mbps} / \mathrm{Km}^{2}$. However, there is no significant difference between those two approaches in terms of coverage extension.

As shown in figure 7-(b), the deployment cost of the WBTC BS based MMR approach is shown to be lower than that of the NBTC BS based MMR system when the RS cost is $10 \%$ or $20 \%$ of the BS cost. However, there is no difference between two approaches when the RS cost is $30 \%$ of BS. Therefore, we could conclude that the WBTC BS-based MMR system is better than the NBTC BS-based MMR system in terms of the coverage extension and deployment cost except for the case when the traffic density is less than $0.4 \mathrm{Mbps} / \mathrm{Km}^{2}$.

\section{Multi-hop system deployment scenario}

In previous two sections, we analyze various MMR WiBro/WiMAX systems for costeffective coverage extension under various topologies and BS sectorizations. From the above analytical results, we can conclude that the multi-hop relay based WiBro/WiMAX system can be deployed in several phases according to the user-traffic density and resulting system performance.

Figure 8 shows a practical deployment scenario of the MMR WiBro/WiMAX system consisting of three deployment scenarios in terms of the user-traffic density and the number 
of tiers. In this scenario we assume that the minimum required data rate per user $128 \mathrm{kbps}$. Phase 1 corresponds to the situation when the traffic density per unit area is less than 1.5 Mbps. In this phase, due to the low traffic density a BS has enough capacity to accommodate many users, and thus the MMR system could have more than 2 tiers to cover large service area. Phase 2 corresponds to the situation when the traffic density is $1.5 \sim 5 \mathrm{Mbps} / \mathrm{Km}^{2}$. In this case, a BS could have 1 or 2 tiers to cover a service area. Phase 3 corresponds to the moderate traffic density situation having more than $5 \mathrm{Mbps} / \mathrm{Km}^{2}$. In this phase, there could be less than 1 tier RSs connected to a BS, and thus RSs are used to extend BS coverage toward a specific direction from a BS or to cover radio shadowing areas.

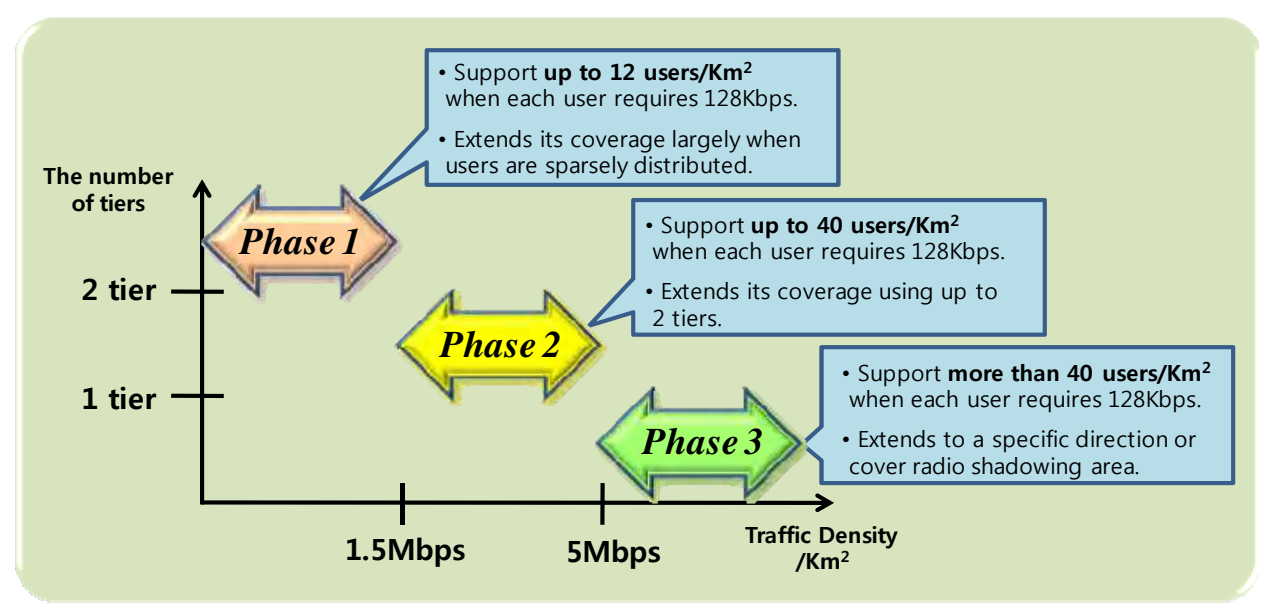

Fig. 8. Deployment Scenario for Multihop-based WiBro/WiMAX systems.

\section{Conclusions}

In this chapter, we first obtained the optimum numbers of BSs and RSs for minimizing total system cost using optimization problem given maximum system capacity of WiBro/ WiMAX systems. We proposed various multi-hop based network topologies using omnidirectional antenna for metropolitan area network and rural area network. We, also, 
proposed multi-hop based network topologies using directional antenna, the NBTC BSbased and the WBTC BS-based MMR systems. Through analytical methods, we obtained the optimal network configuration given traffic density for various topologies and compared the cost with the traditional topology. Finally, we presented practical deployment scenario consisting of three phases depending on user-traffic density and the number of traffic relaying hops. We presented the basic studies of the multi-hop based NBTC BS-based and WBTC BS-based MMR systems without considering interference in this work. However, the WBTC system has generally the bigger interference than the NBTC system. We are planning to investigate and compare the performance of various topologies taking interference into consideration in MMR WiBro / WiMAX systems.

\section{Acknowledgment}

This research was supported by Basic Science Research Program through the National Research Foundation of Korea(NRF) funded by the Ministry of Education, Science and Technology(2010-0025125).

\section{References}

IEEE Standard 802.16e-2005, (2005). IEEE Standard for Local and Metropolitan Area Networks Part 16: Air Interface for Fixed and Mobile Broadband Wireless Access Systems, Feb. 2006.

IEEE 802.16j-2008, (2007). Baseline Document for Draft standard for Local and Metropolitan

Area Networks Part 16: Air Interface for Fixed and Mobile Broadband Wireless Access Systems, June. 2007

WiMAX Forum, http://www.wimaxforum.org

IEEE802.16 TGj PAR, http:/ / grouper.ieee.org/groups/802/16/relay

S. Lee, et al., (2006). "The Wireless Broadband (WiBro) System for Broadband Wireless Internet Services," IEEE Communications Magazine, Vol.44, No.7, (July 2006), pp.106-112, ISSN 0163-6804

Siemens and ETRI, (2007). "IR 1.3 Multi-hop Capabilities of WiMAX," Technical paper, (September 2007).

IEEE 802.16j-06/015, (2007). Guideline documents: Usage Models, IEEE802.16j TG

V. H. MacDonald, (1979). AMPS: The Cellular Concept, Bell System Technical Journal, Vol.58, No.1, (Jan 1979) pp.15-41

Sun-Kuk Noh, (2006). A Study on the WBTC and NBTC for CDMA Mobile Communications Networks, ICCSA 2006, (May 2006) pp.582-590

Dong You Choi, Kwan Houng Lee, (2006). Analysis on the Cell Sectorization Using the Wide-beam and Narrow-beam for CDMA Mobile Communication Channels, International Conference on Computational Intelligence \& Security, Vol.1, (Nov. 2006) pp.882-885

Chang-Hoi Koo, Yong-Woo Chung, Dynamic Cell Coverage Control for Power Saving in IEEE802.16 Mobile Multihop Relay Systems, Systems and Networks Communication 2006, (Oct. 2006) pp.60

IST WINNER II Project, Deliverables-D3.5.1 v1.0, https:/ / www.ist-winner.org 
IST FIREWORKS Project, http:/ / fireworks.intranet.gr

IST ROMANTIK Project, http:/ / www.ist-romantik.org 


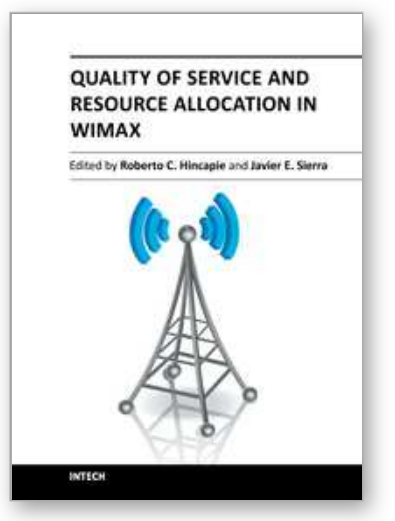

\author{
Quality of Service and Resource Allocation in WiMAX \\ Edited by Dr. Roberto Hincapie
}

ISBN 978-953-307-956-1

Hard cover, 376 pages

Publisher InTech

Published online 03, February, 2012

Published in print edition February, 2012

This book has been prepared to present state of the art on WiMAX Technology. It has been constructed with the support of many researchers around the world, working on resource allocation, quality of service and WiMAX applications. Such many different works on WiMAX, show the great worldwide importance of WiMAX as a wireless broadband access technology. This book is intended for readers interested in resource allocation and quality of service in wireless environments, which is known to be a complex problem. All chapters include both theoretical and technical information, which provides an in depth review of the most recent advances in the field for engineers and researchers, and other readers interested in WiMAX.

\title{
How to reference
}

In order to correctly reference this scholarly work, feel free to copy and paste the following:

Se-Jin Kim, Byung-Bog Lee, Seung-Wan Ryu, Hyong-Woo Lee and Choong-Ho Cho (2012). Cost Effective Coverage Extension in IEEE802.16j Based Mobile WiMAX Systems, Quality of Service and Resource Allocation in WiMAX, Dr. Roberto Hincapie (Ed.), ISBN: 978-953-307-956-1, InTech, Available from:

http://www.intechopen.com/books/quality-of-service-and-resource-allocation-in-wimax/cost-effective-coverageextension-in-ieee802-16j-based-mobile-wimax-systems

\section{INTECH}

open science | open minds

\section{InTech Europe}

University Campus STeP Ri

Slavka Krautzeka 83/A

51000 Rijeka, Croatia

Phone: +385 (51) 770447

Fax: +385 (51) 686166

www.intechopen.com

\section{InTech China}

Unit 405, Office Block, Hotel Equatorial Shanghai

No.65, Yan An Road (West), Shanghai, 200040, China

中国上海市延安西路65号上海国际贵都大饭店办公楼 405 单元

Phone: +86-21-62489820

Fax: $+86-21-62489821$ 
(C) 2012 The Author(s). Licensee IntechOpen. This is an open access article distributed under the terms of the Creative Commons Attribution 3.0 License, which permits unrestricted use, distribution, and reproduction in any medium, provided the original work is properly cited. 